\title{
Factores que influyen en el uso de los protocolos clí- nicos, según la opinión de los fisioterapeutas de los centros de salud de la región de Murcia
}

\author{
F. Medina I Mirapeix, A. B. M eseguer Henarejos, P. J. Saturno Hernán-

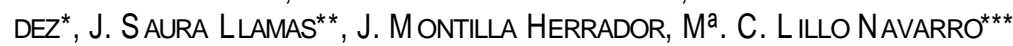 \\ Profesor del Departamento de Fisioterapia. Universidad de Murcia. \\ ${ }^{*}$ Profesor de Medicina Preventiva. Universidad de Murcia. \\ ${ }^{* *}$ Médico de Familia. C.S. El Carmen. Murcia. ${ }^{* *}$ Fisioterapeuta
}

\section{RESUMEN}

Objetivos: averiguar los factores que influyen en el uso de los protocolos de fisioterapia en Atención Primaria según la opinión de los fisioterapeutas de Atención Primaria, las razones de su poco uso y recoger sugerencias para mejora.

Material y métodos: se realizó un estudio de opinión mediante encuesta telefónica. El ámbito de estudio corresponde a 18 Unidades de Fisioterapia del nivel de Atención Primaria de la Comunidad Autónoma de la región de Murcia. Los sujetos de estudio son 21 fisioterapeutas que desarrollan su labor en dichas Unidades.

Se realizó una encuesta telefónica, estructurada, pilotada previamente, administrada por un entrevistador entrenado con dos preguntas abiertas: ¿por qué cree que los fisioterapeutas de su área no utilizan más los protocolos?, y ¿qué sugerencias propondría para que se utilizaran más? El trabajo de campo se realizó en enero de 2000.

Resultados: El grado de respuesta ha sido del 90,9\%. Respecto a la primera pregunta, los fisioterapeutas creen que el poco uso se explicaría por la asistencia mediatizada por criterios médicos, recomendaciones poco actualizadas y poca flexibilidad ante situaciones imprevistas (42,5\% del total de respuestas). Respecto a la segunda pregunta, la sugerencia más señalada ha sido realizar sesiones de consenso para elaborar los protocolos $(13,7 \%)$.

Conclusiones: los fisioterapeutas asumen que los protocolos se usan poco y la clave para utilizarlos más es la mejora de la organización y de los documentos de los protocolos.

Palabras clave: Protocolos clínicos. Encuesta telefónica. Fisioterapeutas Atención Primaria.
Título en inglés

\section{ABSTRACT}

Objectives: to find the factors that affect the use of clinical protocolos in Primary Health Care, in the view of Primary Health Care physiotherapists, the reasons why they are little followed and to bring together suggestions for improvement.

Design: an opinion study was developped though a telephone survey. The studied field are 18 physiotherapy departments at Primary Care in Murcia (autonomus Community). The participants are 21 physiotherapists that work in these depart ments.

Main results: after a pilot study, a structured telephone survey was carried out, administered by a trained interviewer who posed two open questions: why do you think that physiotherapists at your area do no use the protocols more often?, and what suggestions would you make to have the protocols used more? The field-work was done in January 2000.

There was a $90.9 \%$ reply date. To the first ques tion, physiotherapists think that low use could be explained by mediating attendance by doctor's criterions, by few update recommendations and few flexibility before unexpected situations (42.5\% of all replies).

To the second question, the most mentioned suggestion has been to carry out consensus meetings to design protocols (13.7\%).

Conclusions: physiotherapists assumed that the clinical protocols were used little and the key to using them more is to improve the organization and protocol document.

Key words: Clinical protocols. Telephone interviews. Primary Health Care physiotherapists. 


\section{INTRODUCCIÓN}

En España, los fisioterapeutas se incorporaron a la Atención Primaria en 1990 como profesionales de apoyo a los Equipos de Atención Primaria ${ }^{1}$. Desde entonces se vienen desarrollando protocolos en los centros de salud con fisioterapia ${ }^{2}$, y más aún a partir de que se publicara en 1991 la normativa sobre la ordenación de las actividades de los fisioterapeutas de área ${ }^{3}$, que insistió en la necesidad de una atención protocolizada.

Los protocolos o guías de práctica clínica ${ }^{4,5}$ son actualmente parte esencial del proceso de mejora de la calidad clínica ${ }^{6}$, existiendo una cierta evidencia empírica de su utilidad y efecto positivo en el coste de la asistencia para eliminar actuaciones superfluas ${ }^{7}$. La importancia de los protocolos radica en que son herramientas que facilitan la toma de decisiones al clínico, le ayudan a luchar contra la incertidumbre y a disminuir la variabilidad de la práctica clínica. Sin embargo, para que esto sea posible es necesario que dicho instrumento tenga una mínima calidad.

La investigación sobre protocolos clínicos es actualmente un campo de primer orden ${ }^{8,9}$, lo que ha dado lugar a interesantes aportaciones en este terreno ${ }^{4,5,10-12}$. Una importante ha sido la necesidad de evaluar los protocolos en cuatro dimensiones ${ }^{13}$ : la calidad formal (entendida como los requisitos estructurales exigibles a un protocolo), su utilización, la calidad de los contenidos específicos del aspecto protocolizado y su efectividad, es decir, los resultados que haya podido generar.

Con relación a la calidad formal del diseño nuestro grupo en base a criterios elaborados a partir de la línea de investigación en la que se viene trabajando para la evaluación de los protocolos clíni$\cos ^{14,15}$, evidenció una baja calidad global de los protocolos de Fisioterapia de Atención Primaria en un reciente estudio con protocolos procedentes de toda España elaborados mayoritariamente por médicos y fisioterapeutas ${ }^{16}$.

Con relación a la utilización de los protocolos existe una cierta controversia sobre cómo hacer para que realmente se usen, sean adoptados por los profesionales y funcionen ${ }^{12}$ Para aportar un poco de claridad a esta controversia consideramos que conviene considerar las características que los profesio nales les piden a los documentos de los protocolos para que los usen. Un primer acercamiento posible es la realización de una encuesta de opinión dirigida a los fisioterapeutas que utilizan los protocolos.

Los objetivos del presente estudio son establecer y definir las características que los fisioterapeutas de Atención Primaria de la región de Murcia exigen a los protocolos clínicos (expectativas del cliente interno), los factores que influyen en el uso de los mismos (razones para no utilizarlos) y proponer medidas para que se utilicen con más frecuencia.

\section{MATERIAL Y MÉTODOS}

Se trata de un estudio de opinión, descriptivo. El ámbito de estudio corresponde a las Unidades de Fisioterapia del nivel de Atención Primaria de la Comunidad Autónoma de la región de Murcia; en total, 18 Unidades de Fisioterapia distribuidas en tres Áreas de Salud: Cartagena (con siete), Lorca (con cuatro) y Murcia (con siete).

Los sujetos de estudio son todos los fisioterapeutas de Atención Primaria que desarrollan su labor en dichas Unidades, en total 22 personas. A todo el universo se ha realizado una entrevista telefónica personal. Se han excluido del estudio aquellas personas que por cualquier motivo no se les localizara o se negaran a colaborar en la encuesta. Está previsto completar esta información con la realización de grupos focales con los mismos fisioterapeutas, pero estos datos no se incluyen aquí al no estar aún disponibles.

El cuestionario utilizado en la entrevista lo elaboró el equipo de investigación a partir del utilizado en una experiencia similar y previa realizada por otros miembros del equipo con coordinadores médicos y responsables de enfermería ${ }^{17}$. Las dos preguntas utilizadas fueron abiertas: ¿por qué cree que los fisioterapeutas de su Área de Salud no utilizan más los protocolos?, y ¿qué sugerencias propondría para que se utilizaran más?

La encuesta telefónica se ha realizado por un entrevistador previamente entrenado para este estudio. También se ha realizado un pilotaje previo aplicándolo a diversos fisioterapeutas de Atención Primaria ajenos al estudio, procedentes de otras Comunidades Autónomas. Este pilotaje sirvió para poder mejorar la sencillez y comprensión de las preguntas, quedando formuladas del modo que se ha expuesto en el párrafo anterior.

El trabajo de campo se ha realizado durante el mes de enero del 2000. Para realizar la entrevista se han utilizado las técnicas de entrevista telefónica, tal como se describen en la bibliografía ${ }^{18,19}$. En la primera llamada se le explicaba el motivo de la misma y se le solicitaba un tiempo aproximado de 3 minutos para contestar al tiempo que se le invitaba que eligiera contestar en el momento o en una segunda llamada realizada en la hora o día que el fisioterapeuta predeterminara.

Cada encuestado podía proporcionar más de una respuesta a cada una de las dos preguntas. Para recoger las respuestas se diseñó una plantilla que incluía, además de opciones abiertas, opciones con las diferentes categorías o motivos posibles identificados a través de una previa revisión de la literatura científica sobre factores que influyen en el uso de los protocolos, todo ello con el fin de asegurar una cierta transferencia y comparabilidad de los resultados del estudio. 
Inicialmente las preguntas se recogían textualmente en la planilla. No obstante la clasificación final de las respuestas se ha realizado a partir de la discusión del grupo de investigación empleando la técnica de análisis del "contenido de variables cualitativas". El conjunto de respuestas se ha clasificado en diferentes motivos y éstos en bloques. Se ha calculado la tasa de respuesta por bloque y la media de respuestas por motivo identificado.

\section{RESULTADOS}

La proporción de encuestas telefónicas completas realizadas ha sido de 20 , respecto a las 22 posibles, que constituye el total de la población $(90,9 \%)$. Sólo dos fisioterapeutas no contestaron la encuesta: uno por no querer colaborar y otro por no haber podido establecer contacto con él.

Respuestas a la primera pregunta (¿por qué cree que los profesionales de su Área de Salud no utilizan más los protocolos?). Esta pregunta la contestaron los 20 que aceptaron participar en la encuesta. Ninguno de ellos contestó que los protocolos ya se utilizaran mucho y que por tanto la pregunta era improcedente. El conjunto de los encuestados dieron lugar a 40 respuestas, lo que equivale a una media de 2 respuestas por fisioterapeuta.

Las respuestas obtenidas se agrupan en un total de 13 motivos diferentes que explican el poco uso de los protocolos (Tabla I). Los motivos más señalados son que los protocolos incluyen habitual-

\begin{tabular}{|c|c|c|}
\hline \multicolumn{3}{|c|}{$\begin{array}{c}\text { MOTIVOS POR LOS QUE NO SE UTILIZAN MÁS LOS } \\
\text { PROTOCOLOS }\end{array}$} \\
\hline MOTIVOS & \multicolumn{2}{|c|}{ № DE RESPUESTA\% } \\
\hline 1. Asistencia con criterios de especializada & 6 & 15 \\
\hline 2. No tienen los protocolos de su área & 4 & 10 \\
\hline 3. Falta de tiempo & 3 & 7,5 \\
\hline 4. Falta de recursos materiales & 3 & 7,5 \\
\hline 5. Exceso de demanda & 2 & 5 \\
\hline 6. Apoyo insuficiente desde Gerencia & 1 & 2,5 \\
\hline 7. Incluye recomendaciones poco actualizadas & 6 & 15 \\
\hline 8. Poco flexibles ante situaciones imprevistas & 5 & 12,5 \\
\hline 9. Poco útiles, poco prácticos & 4 & 10 \\
\hline 10. Pobre expectativa de resultados & 2 & 5 \\
\hline 11. Poca identificación o familiaridad con protocolo & 2 & 5 \\
\hline 12. Insuficiente motivación & 1 & 2,5 \\
\hline 13. Falta de formación para elaborarlos & 1 & 2,5 \\
\hline $\begin{array}{l}\text { Total de respuestas } \\
\text { Media motivos por fisioterapeuta }\end{array}$ & $\begin{array}{c}40 \\
2\end{array}$ & 100 \\
\hline
\end{tabular}

mente recomendaciones poco actualizadas (en el $15 \%)$, su poca flexibilidad ante situaciones imprevistas $(12,5 \%)$ y tener la asistencia mediatizada por los criterios de médicos especialistas en rehabilitación en lugar de por protocolos (15\%). La falta de formación para elaborarlos es uno de los motivos menos señalados $(2,5 \%)$; destaca sobre todo por cuanto que dos de los tres motivos más señalados (poca actualización y flexibilidad) hacen referencia a problemas con los documentos de los protocolos.

Los 13 motivos identificados se han agrupado en tres bloques, según se trate de problemas de organización y de funcionamiento (motivos del 1 al 6), problemas de los documentos de los protocolos (del 7 al 9) o de problemas de las personas que los utilizan (del 10 al 13). El bloque de organización es el que tiene mayor número de motivos diferentes (el $46 \%$ ).

En la tabla II se puede observar cómo el mayor número de respuestas totales los reciben los bloques de organización y funcionamiento, y el de problemas con los documentos de los protocolos (en el 47,5 y el 37,5\%, respectivamente), no obstante son los motivos del bloque de organización y funcionamiento los que presentan una mayor media de respuestas 5 . El bloque de problemas del personal que los utiliza es el que presenta un menor número de respuestas.

Respuestas a la segunda pregunta (¿qué sugerencias propondría para que se utilizarán más?). Esta pregunta no la contestaron 5 (25\%) de los 20 que aceptaron participar en la encuesta por no saber qué decir. El resto de encuestados dieron lugar a 29 respuestas, lo que equivale a una media de 1,9 respuestas por fisioterapeuta que contesta.

Las respuestas obtenidas se agrupan en un total de 15 sugerencias diferentes (Tabla III). La sugerencia más señalada es que se realicen sesiones de consenso para elaborarlos $(13,7 \%)$.

Las 15 sugerencias identificadas se han agrupado en tres bloques, según se trate de sugerencias para mejorar la organización y el funcionamiento (sugerencias de la 1 a la 8), para mejorar los documentos de los protocolos (de la 9 a la 12) o para otros aspectos (de la 13 a la 15). El bloque de sugerencias para la organización y funcionamiento es el que tiene mayor número de sugerencias diferentes (el 53,3\%)

En la tabla IV se puede observar cómo el número de respuestas totales que reciben los bloques de organización y funcionamiento, y el de problemas con los documentos de los protocolos son los que obtienen un mayor número de respuestas (el 48,3 y el 41,4\%, respectivamente), sin embargo son los motivos del bloque de sugerencias para la mejora de los documentos de los protocolos los que presentan una mayor media de respuesta ${ }^{3}$. 


\section{Tabla II}

MOTIVOS Y RESPUESTAS AGRUPADOS POR BLOQUES Y TASA DE NÚMERO DE RESPUESTAS POR LOS MOTIVOS DE CADA BLOQUE

\begin{tabular}{lccccc}
\hline \multirow{2}{*}{ Bloques de motivos } & \multicolumn{2}{c}{ Motivos } & \multicolumn{2}{c}{ Respuestas } & Media repuestas/ motivo \\
& $n$ & $\%$ & $n$ & $\%$ & 3,17 \\
\hline Problemas de organización y funcionamiento & 6 & 46 & 19 & 47,5 & 5 \\
Problemas de los documentos de los protocolos & 3 & 23 & 15 & 37,5 & 1,5 \\
Problemas del personal que los utiliza & 4 & 31 & 6 & 15 & 0,3 \\
\hline Total & 13 & 100 & 40 & 100 & \\
\hline
\end{tabular}

\begin{tabular}{|c|c|c|}
\hline \multicolumn{3}{|c|}{ SUGERENCIAS PARA QUE LOS PROTOCOLOS SE } \\
\hline $\begin{array}{l}\text { SUGERENCIAS } \\
\%\end{array}$ & SPL & ESTAS \\
\hline 1. Más recursos materiales & 3 & 10,3 \\
\hline 2. Proporcionar tiempo & 3 & 10,3 \\
\hline 3. Disminuir la demanda & 2 & 7,1 \\
\hline 4. Instituirlos en todos los centros de salud & 2 & 7,1 \\
\hline 5. Tener más sesiones clínicas & 1 & 3,4 \\
\hline 6. Tratamiento no mediado por el rehabilitador & 1 & 3,4 \\
\hline 7. Divulgación de los protocolos en EAP & 1 & 3,4 \\
\hline 8. Coordinación con Atención Especializada & 1 & 3,4 \\
\hline 9. Sesiones de consenso para elaborarlos & 4 & 13,7 \\
\hline 10. Actualizarlos por quienes lo van a utilizar & 3 & 10,3 \\
\hline 11. Más flexibles & 3 & 10,3 \\
\hline 12. Simplificarlos, sencillez de uso & 2 & 7,1 \\
\hline 13. Más control de sus resultados & 1 & 3,4 \\
\hline 14. Mejor conocimiento de la metodología de AP & 1 & 3,4 \\
\hline 15. Convencer a la gente & 1 & 3,4 \\
\hline Suma de sugerencias & 29 & 100 \\
\hline
\end{tabular}

\section{DISCUSIÓN}

La tasa de respuesta ha sido muy elevada ya que sólo una persona se negó a colaborar. La proporción de respuestas a las dos preguntas se puede considerar muy alta $(87,5 \%)$ con relación a experiencias previas similares con coordinadores médicos y responsables de enfermería ${ }^{17}$.

A pesar de que en el presente estudio la contestación a la segunda pregunta sea inferior a la primera, la media de respuestas por fisioterapeuta es muy similar para ambas preguntas $(2$ y 1,9$)$. Aunque es algo superior a la proporcionada por médicos y enfermeras ${ }^{17}$, sigue siendo algo escasa. Este dato podría significar un escaso interés por el tema, desconocimiento o dificultad para identificar problemas y proponer soluciones en periodo de tiempo tan corto como el que dura una encuesta telefónica.

Los posibles sesgos dependientes de la validez y utilidad de las técnicas y de la metodología elegida son probablemente bajos por ser unas técnicas sencillas y consistentes ${ }^{18-20}$. El posible sesgo en la recogida de la información lo consideramos mínimo al realizarla un entrevistador previamente entrenado, con una entrevista estructurada y reco-

\section{Tabla IV}

\section{SUGERENCIAS Y RESPUESTAS AGRUPADOS POR BLOQUES Y TASA DEL NÚMERO DE REPUESTAS POR LAS SUGERENCIAS}

\begin{tabular}{|c|c|c|c|c|c|}
\hline \multirow[t]{2}{*}{ Bloques de sugerencias } & \multicolumn{2}{|c|}{ Sugerencias } & \multicolumn{2}{|c|}{ Respuestas } & \multirow{2}{*}{$\begin{array}{c}\text { Media de repuestas } \\
\text { / sugerencia }\end{array}$} \\
\hline & $n$ & $\%$ & $n$ & $\%$ & \\
\hline Mejora de la organización y funcionamiento & 8 & 53,3 & 14 & 48,3 & 1,75 \\
\hline Mejora de los documentos de los protocolos & 4 & 26,7 & 12 & 41,4 & 3 \\
\hline Otras & 3 & 20 & 3 & 10,3 & 1 \\
\hline Total & 15 & 100 & 29 & 100 & 1,93 \\
\hline
\end{tabular}


gida en una planilla. El sesgo de interpretación por la presentación de los datos (categorización de las respuestas), y el no incluir la transcripción literal de todos los discursos (por la necesaria brevedad), creemos que han sido parcialmente obviados por el proceso de discusión establecido en el grupo de investigación.

De los resultados obtenidos en esta encuesta se puede deducir que los fisioterapeutas aceptan que los protocolos no se utilizan lo suficiente y que se deben desarrollar medidas que mejoren su uso.

Con relación a los problemas detectados, hay bastante acuerdo en señalar que la asistencia mediatizada por los criterios de médicos especialistas y la falta de actualización son los factores más limitantes. La "flexibilidad", el "ser poco prácticos" o el "no disponer de protocolos" son otros problemas importantes que justifican el poco uso. Los problemas más importantes están relacionados con la organización y el funcionamiento, y con los documentos de los protocolos. La mayor media de respuestas ofrecidas para este último tipo de problema indica que existe mayor acuerdo en cuáles son los motivos procedentes de los documentos que afectan negativamente a su uso que en los procedentes de la organización y el funcionamiento o el personal que los utiliza.

A pesar de que existan muchas respuestas relacionadas con los defectos de los protocolos, los fisioterapeutas no perciben como una posible causa de ellos una falta de formación específica. Quizás sea por desconocimiento de que existe toda una serie de publicaciones relacionadas con la metodología de elaboración y puesta en marcha de los protocolos. Probablemente crean que la capacidad de elaborar protocolos clínicos se les presupone por el hecho de ser profesionales sanitarios.
Con relación a las sugerencias, las propuestas son más variadas aunque también siguen teniendo mucha importancia las medidas para mejorar los documentos de los protocolos. Las principales sugerencias relativas son el "consenso", la "actualización" y la mayor "flexibilidad y sencillez de uso", en esencia muy parecidas a las señaladas por coordinadores médicos y de enfermería ${ }^{17}$. El bloque de sugerencias relativas a la mejora de la organización presenta un número elevado de respuestas, pero presenta una mayor dispersión (al igual que sucede para los motivos).

La mayoría de las sugerencias que proponen los fisioterapeutas para mejorar los protocolos ya fueron identificadas por el IOM (Institute of Medicine) como criterios para el diseño y elaboración de los protocolos clínicos ${ }^{4,5}$. Sin embargo, otros criterios relevantes tal y como la aplicabilidad clínica y la validez o la fiabilidad no han sido mencionados por los fisioterapeutas. Esto puede traducir que los encuestados dan mayor importancia a las sugerencias aquí recogidas (sencillez, flexibilidad, etc.) y que en general tienen relación con la facilidad de uso en la consulta, que a otras opciones; pero también podría significar un desconocimiento del tema.

\section{Bibliografía}

1. Resolución, de 19 de febrero, del Ministerio de Sanidad y Consumo. BOE de 27 de febrero 1990.

2. Medina i Mirapeix F, Torres Vaquero Al, Bonilla Solís R, Montilla Herrador J, Escolar Reina P, Valera Garrido JF. Características de los protocolos de fisioterapia elaborados en Atención Primaria durante el periodo 1990-1996. Centro de Salud 1999; 7 (11): $697-$ 702.

3. Dirección General del Insalud. Circular 4/91 sobre Ordenación de las actividades y funciones del Fisioterapueta de Área en Atención Primaria. Madrid 1991.

4. Field MJ, Lohr KN. Clinical practice guidelines. Direc- tions for a new program. Washington DC: National Academy Press, 1990.

5. Field MJ, Lohr KN. Guideline for clinical practice From development to use. Washington DC: National Academy Press, 1992.

6. Schoenbaum SC, Gottbieb LK. Algorithm based improvement of clinical quality. Br Med J 1990; 310: 1374-6.

7. Orient JM, Kettel LJ, Sox HC. The effect of algoritms on the cost and quality of patient care. Medical Care 1983; 21 (2): 157-67.

8. Agency for Health Care Policy and Research. Guideline definition and attributes. Rockville: Department of 
Health and Human Services, 1990.

9. Bolster CJ. Quality research iniciatives. Top Health Care Financ 1991; 18 (2): 7-11.

10. Eddy DM. Practice policies: where do they come from? JAMA 1990; 263 (9): 1265-75.

11. Brook RH. Practice guidelines and practicing medicine; are they compatible? JAMA 1989; 262 (21): $3027-$ 30.

12. Lomas J, Anderson GM, Dominick-Pierre K. Do practice guidelines guide practice? The effect of a consensus statement on the practice of physicians. N Engl J Med 1989; 321: 1036-311.

13. Saturno PJ. Evaluación de los protocolos o guías de práctica. En: Saturno PJ. Métodos y herramientas para el diseño de la calidad. Protocolización de actividades clínicas y diseño de procesos (Módulo V). Master en gestión de la calidad en los servicios de salud. Universidad de Murcia, 1999.

14. Saura Llamas J, Saturno Hernández PJ y Grupo de Evaluación y Mejora de los Protocolos Clínicos. Protocolos clínicos: ¿cómo se construyen? Propuesta de un modelo para su diseño y elaboración. Aten Primaria 1996; 18: 91-6.

15. Saura Llamas J, Saturno Hernández PJ. Evaluación y mejora del diseño de los protocolos clínicos. Aten Primaria 1994; 13: 355-61.

16. Medina F, Torres AI, Saura J, Saturno P y otros miembros del equipo de evaluación de los protocolos. Evaluación de la calidad estructural de los protocolos clínicos de fisioterapia elaborados en atención primaria. Aten Prim 1999; 23: 520-5.

17. Saura J, Saturno PJ, Gaona JM, Romero JR, Gónzalez M y Grupo de Investigación. Factores que influyen en el uso de los protocolos clínicos, según la opinión 